\title{
Corrosión en sales fundidas de un acero recubierto mediante rociado térmico por llama
}

\author{
Hot corrosion of coated steel by flame spray
}

\author{
José L. Marulanda $\mathbf{A}^{1}$, José L.Tristancho $\mathbf{R}^{2}$, Aduljay Remolina $\mathbf{M}^{3}$. \\ ${ }^{1}$ Doctor en Ciencias Químicas, Facultad de Ingeniería Mecánica, Universidad Tecnológica de Pereira, \\ Grupo de Investigación en Materiales Avanzados GIMAV - UTP, Pereira, Colombia: jlmarulanda@utp.edu.co \\ ${ }^{2}$ Doctor en Ciencias de los Materiales, Facultad de Ingeniería Mecánica, Universidad Tecnológica de Pereira, \\ Grupo de Investigación en Materiales Avanzados GIMAV - UTP, Pereira, Colombia: josetris@utp.edu.co \\ ${ }^{3}$ Doctor en Ciencia de Materiales, Facultad de Ingeniería Mecánica, Universidad Pontificia Bolivariana, \\ Grupo de Investigación en Desarrollo Tecnológico, Bucaramanga, Colombia.
}

Recibido 9/02/14, Aceptado 6/03/2014

\section{RESUMEN}

Se evaluó la velocidad de corrosión de un acero laminado en caliente, recubierto con una aleación $\mathrm{Ni}$-Cr, en presencia de sales fundidas con una composición de $80 \%$ pentóxido de vanadio $\left(V_{2} \mathrm{O}_{5}\right)$ y $20 \%$ Sulfato de sodio $\left(\mathrm{Na}_{2} \mathrm{SO}_{4}\right)$, mediante la técnica de pérdida de peso. La evaluación de la velocidad de corrosión se realizó variando la temperatura entre $650-750{ }^{\circ} \mathrm{C}$, durante 1,7 y 22 horas. El recubrimiento de $\mathrm{Ni}-\mathrm{Cr}$ obtenido por la técnica de rociado térmico produce una capa que reduce la velocidad de corrosión, sin embargo es susceptible al ataque corrosivo de las sales fundidas a las que se encuentra expuesto dicho recubrimiento. Encontramos que la resistencia a la corrosión del recubrimiento depende de la naturaleza de la capa de óxido, los componentes de la aleación y los constituyentes de las sales.

Palabras clave: Corrosión, Sales fundidas, Óxidos, Rociado térmico, Recubrimientos.

\begin{abstract}
We assessed the corrosion rate of hot-rolled steel coated with a Ni-Cr alloy in the presence of molten salts with a composition of $80 \%$ vanadium pentoxide $\left(\mathrm{V}_{2} \mathrm{O}_{5}\right)$ and $20 \%$ sodium sulfate $\left(\mathrm{Na}_{2} \mathrm{SO}_{4}\right)$ by weight loss technique. Evaluating the corrosion rate was done by varying the temperature between $650-750{ }^{\circ} \mathrm{C}$ for 1,7 and 22 hours. The NiCr coating obtained by thermal spraying technique produces a coating that reduces the corrosion rate, however, is susceptible to corrosive attack by the molten salts to which the coating is exposed. Found that the corrosion resistance of the coating depends on the nature of the oxide layer, the components of the alloy constituents and salts.
\end{abstract}

Keywords: Corrosion, Molten salts, Oxides, Thermal spraying, Coatings.

\section{INTRODUCCIÓN}

La degradación de metales y aleaciones por corrosión en sales fundidas ha sido reconocida como un problema grave para muchas aplicaciones, tales como: calderas, motores de combustión interna, turbinas de gas, incineradores de residuos industriales entre otras. Debido al agotamiento de los combustibles y por razones económicas, se está utilizando aceite residual junto con carbón en los sistemas de generación de energía, los cuales contienen sodio, vanadio y azufre como impurezas. Durante la combustión, el $\mathrm{Na}$ y $\mathrm{S}$ reaccionan entre sí y para formar $\mathrm{Na}_{2} \mathrm{SO}_{4}$, además, el vanadio reacciona con el oxígeno para formar vanadatos complejos $V_{2} \mathrm{O}_{5}$ entre otros compuestos.Estos compuestos se depositan en la superficie de los materiales, induciendo la corrosión, ya que destruyen las capas de óxido protectoras que forman los materiales durante el funcionamiento [1]. 
Además, para mitigar el cambio climático global, se ha aumentado el uso de energía sostenible, incluyendo la biomasa y combustibles derivados de residuos, para reemplazar a los combustibles fósiles. Una consecuencia de esta tendencia es la previsión de una mayor incidencia de la corrosión en sales fundidas en calderas, turbinas, incineradores entre otros equipos, debido al aumento de impurezas en la combustión, las cuales forman depósitos superficiales fundidos que inician el proceso de corrosión [2].

En los intentos de mitigar los procesos corrosivos y reducir los costos de mantenimiento de estos componentes, se ha aumentado el interés por desarrollar recubrimientos con alto contenido de cromo o níquel-cromo, que protejan los sustratos de ambientes agresivos como es el caso de las sales fundidas, ya que forman una capa de $\mathrm{Cr}_{2} \mathrm{O}_{3}$ [3]. Los recubrimientos obtenidos por la tecnología del rociado térmico pueden ser rentables para proteger la superficie del sustrato contra el desgaste o la corrosión. Otras aplicaciones de los recubrimientos obtenidos por rociado térmicos comprenden la restauración de medidas, obtener tolerancias precisas, así como modificar las propiedades térmicas y eléctricas. Además, el recubrimiento aplicado por la tecnología del rociado térmico tiende a prolongar la vida útil de los equipos, al obtener altos rendimientos de producción durante más tiempo y reducir el número de fallos. La avanzada tecnología del rociado térmico, provee uno de los medios más eficaces que se hayan desarrollado para combatir el desgaste prematuro, adelantándose a la falla o al momento en que el equipo o elemento deja de trabajar en sus condiciones óptimas [4].

\section{REVESTIMIENTOS POR ROCIADO TÉRMICO}

La tecnología del rociado térmico tiene lugar en la ingeniería de superficie. Algunas ventajas incluyen la facilidad de aplicación, poco impacto ambiental, bajo costo. Estos recubrimientos puede ser la etapa final en un sistema de producción o puede constituir una técnica de restauración de la superficie. Las aplicaciones incluyen la protección de una gran variedad de materiales de ingeniería, para aumentar la vida de los componentes de máquina y/o equipos, cuya superficie se somete a condiciones severas de trabajo. Algunas aplicaciones son la reparación de ejes desgastados y la protección contra la corrosión entre otras. Esta tecnología permite altas velocidades de deposición y puede producir recubrimientos gruesos en los componentes de gran tamaño y de geometría compleja $[5,6]$.

Cada una de las aleaciones de metales en polvo es un compuesto especializado que permite lograr altos niveles de rendimiento. El tamaño controlado de las partículas de cada aleación asegura una alta eficiencia del rociado térmico por polvos [7]. Para la aplicación de las capas rociadas térmicamente se usa una antorcha (Pistola) para calentar el material en forma del polvo o de alambre, y se lleva a un estado fundido o semi-fundido y con el uso de un gas se propulsa el material de aporte, que va a formar el recubrimiento hacia el substrato, creando una superficie totalmente nueva. El material de la capa puede ser un solo elemento, una aleación o compuestos con características físicas y químicas únicas, que en la mayoría de los casos, solo son alcanzables con este proceso de rociado térmico. Las capas rociadas térmicamente son un método altamente rentable y sirve para agregar características y calidades superiores de funcionamiento a una superficie dada [8]. La figura 1 ilustra esquemáticamente, la sección transversal de un revestimiento rociado térmicamente.

Figura 1. Sección transversal de un revestimiento rociado, mostrando la estructura laminar de óxidos e inclusiones [4]. Figure 1. Cross section of a spray coating, showing laminar structure of oxides and inclusions [4].

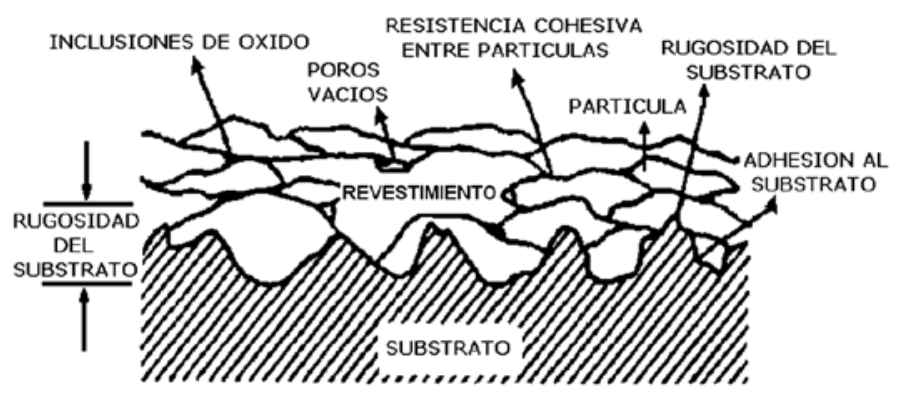

En términos muy simples, el recubrimiento rociado térmicamente comprende el calentamiento del material, en forma de polvo o de alambre, hasta obtener una forma fundida o semi-fundida. Este material se transporta mediante una corriente de gas o de aire comprimido para su depósito, creando una estructura en la superficie en un determinado substrato. Este proceso es de naturaleza sinergética, ya que, las diversas variables y componentes que existen, producen un efecto mayor cuando actúan juntos, que cuando son considerados individualmente. En estos procesos el material de aporte que se alimenta, se funde y se proyecta sobre la superficie previamente preparada, en donde estas partículas chocan con la superficie del substrato, aplanándose y formando finas partículas lenticulares que se solidifican, conformándose y adhiriéndose a las irregularidades de la superficie y entre sí, formando una estructura laminar [5].

En los procesos de rociado térmico ocurren dos etapas distintas: (1) Atomización y (2) deposición. En la atomización tiene lugar el rompimiento del material líquido fundido en góticas y en la deposición se presentan dos momentos distintos: (2.1) donde la gota está viajando e interactúa con el gas de atomización y (2.2) donde las góticas impactan e interactúan con el substrato [10] 


\section{CORROSIÓN Y OXIDACIÓN}

La corrosión es el resultado destructivo de un material por una reacción química o electroquímica entre un metal o aleación y su medio ambiente, en donde los átomos metálicos pasan a compuestos químicos o minerales, ya que la corrosión es la tendencia que tienen los metales a volver al estado combinado, es decir, al mismo estado en que se encontraban en la naturaleza, que es, en términos termodinámicos, el estado más estable, también se puede afirmar que la corrosión electroquímica es un proceso espontáneo donde existe una zona anódica (que sufre la corrosión), una zona catódica (la cual está protegida) y un electrolito, y es imprescindible la existencia de estos tres elementos, además de una buena unión eléctrica entre ánodos y cátodos, para que este tipo de corrosión pueda tener lugar. La corrosión más frecuente es de naturaleza electroquímica y resulta de la formación de muchas zonas anódicas y catódicas sobre la superficie metálica $[9,11]$.

\section{- Corrosión por sales fundidas}

La corrosión acelerada a alta temperatura, suele ocurrir sobre los materiales metálicos cuando estos están en contacto con una película de sal fundida en un ambiente oxidante; generalmente, esto ocurre en los sobre calentadores y recalentadores de las calderas, donde se genera la formación de cenizas durante los procesos de combustión, las cuales, tienen una alta concentración de compuestos de vanadio, sodio y azufre, principalmente como complejos de $\mathrm{Na}_{2} \mathrm{SO}_{4}, \mathrm{~V}_{2} \mathrm{O}_{5}$ y mezclas de sodio - vanadio. La corrosión de estos sistemas de combustión, es el principal obstáculo en la eficiencia térmica de las plantas que queman combustibles fósiles $[12,13]$.

Las impurezas más agresivas en un proceso de combustión, son el vanadio y el azufre; el vanadio del combustible se transforma durante el proceso en $V_{2} \mathrm{O}_{5}$ y el azufre en la ceniza está en forma de sulfatos alcalinos. Esta clase de compuestos $\left(\mathrm{Na}_{2} \mathrm{SO}_{4}\right.$ y $\left.\mathrm{V}_{2} \mathrm{O}_{5}\right)$ forman un sistema binario que sufre una reacción eutéctica a temperatura relativamente baja (menor de $600{ }^{\circ} \mathrm{C}$ ), la cual causa fusión, formando una capa estable de electrolito sobre la superficie del metal [14]. Ver figura 2.

El fenómeno corrosivo depende exponencialmente de la temperatura de la superficie externa del metal, siendo mínimo el daño hasta $590{ }^{\circ} \mathrm{C}$, normal hasta $600{ }^{\circ} \mathrm{C}$, severo hasta $625^{\circ} \mathrm{C}$ y de carácter catastrófico por encima de esta temperatura; además, las irregularidades en el mecanismo corrosivo pueden inducir fallas prematuras o catastróficas a corto tiempo.
Figura 2. Diagrama de fases del sistema $\mathrm{V}_{2} \mathrm{O}_{5}-\mathrm{Na}_{2} \mathrm{O}$ [12]. Figure 2. Phase diagram of $\mathrm{V}_{2} \mathrm{O}_{5}-\mathrm{Na}_{2} \mathrm{O}$ system [12]

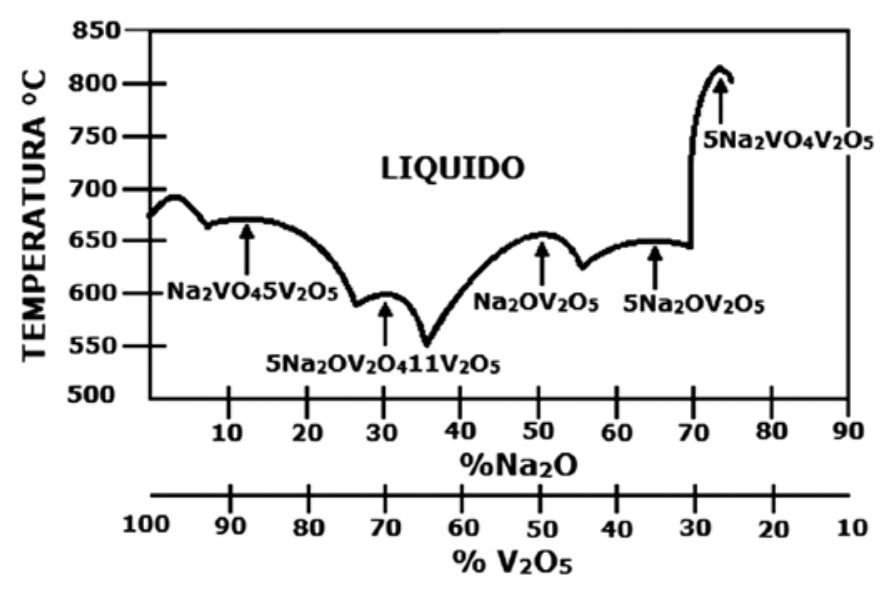

El ataque corrosivo se acelera cuando los componentes del sistema alcanzan las temperaturas de fusión del depósito salino; el cual ejerce una acción fundente sobre el óxido protector, deteriorándolo por disolución y favoreciendo el transporte de especies oxidantes hacia el metal y de iones metálicos hacia la sal, iniciando así la etapa de propagación o ataque acelerado. En la interfaz metal-óxido, se presenta el desprendimiento de los iones metálicos y su consecuente formación de huecos de electrones; esto hace, que en la interfaz óxido-sal, el desprendimiento y la disolución de la capa protectora sea acelerada, y a medida que avanza hacia el seno de la película de sal fundida, y en cercanía de la interfaz sal-gas, este óxido re-precipita como un óxido poroso no protector. [14] En la figura 3, se observa un esquema del posible mecanismo de ataque.

La deposición de la película de sal fundida sobre la superficie metálica se produce por deposición química cuando la presión de vapor del $\mathrm{Na}_{2} \mathrm{SO}_{4}$ dentro de la fase gaseosa, a la temperatura del sustrato metálico, excede la presión parcial de equilibrio; o por deposición física cuando varias sales, sólidas o líquidas, pueden desprenderse de un componente corriente arriba (Ejemplo: filtro de aire) y se adhiere por impacto al sustrato metálico. La corrosión en caliente o a alta temperatura se divide en dos amplias categorías a saber:

- Corrosión en caliente tipo I: denominada HTHC, este mecanismo de corrosión se observó en el intervalo de temperatura comprendido entre 750 y $950{ }^{\circ} \mathrm{C}$, cuando la película de sal está completamente líquida, presenta velocidad máxima de corrosión a $\operatorname{los} 900^{\circ} \mathrm{C}$. Una microestructura típica muestra la formación de sulfuros y un agotamiento o disminución de los componentes activos en el sustrato metálico. 
Figura 3. Mecanismo de precipitación de óxidos porosos por un gradiente de solubilidad negativo y sus compuestos en la película de sal fundida [14].

Figure 3. Precipitation mechanism of porous oxides by a negative solubility gradient and its compounds in molten salt film [14].

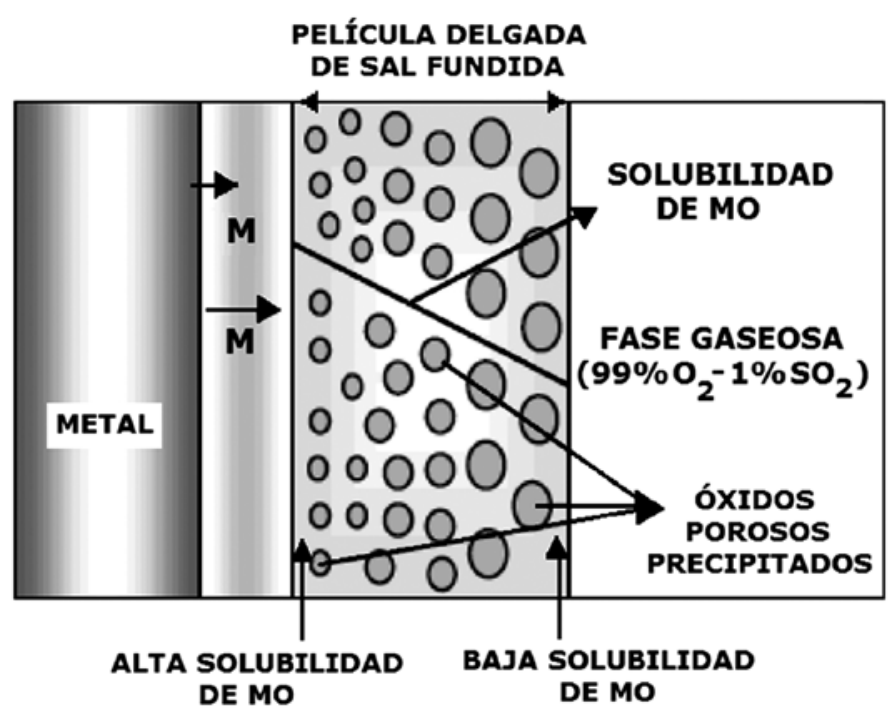

- Corrosión en caliente tipo II: Denominada LTHC, se ubica entre $\operatorname{los} 650^{\circ} \mathrm{C}$ y los $750^{\circ} \mathrm{C}$, donde la fase líquida de la sal es formada únicamente por la disolución de varios productos de corrosión, ocurre por debajo del punto de fusión del $\mathrm{Na}_{2} \mathrm{SO}_{4}$ puro y se caracteriza por un ataque no uniforme en forma de huecos con una pequeña formación de sulfuros cerca de la interfaz metal - óxido y una disminución de $\mathrm{Cr}$ o $\mathrm{Al}$ en el sustrato de la aleación. Ver figura 4.

Figura 4. Velocidad de corrosión en función de la temperatura ilustrando los efectos de la corrosión tipo I y II [13]. Figure 4. Corrosion rates in function of temperature to illustrate the effects of type I and II [13].

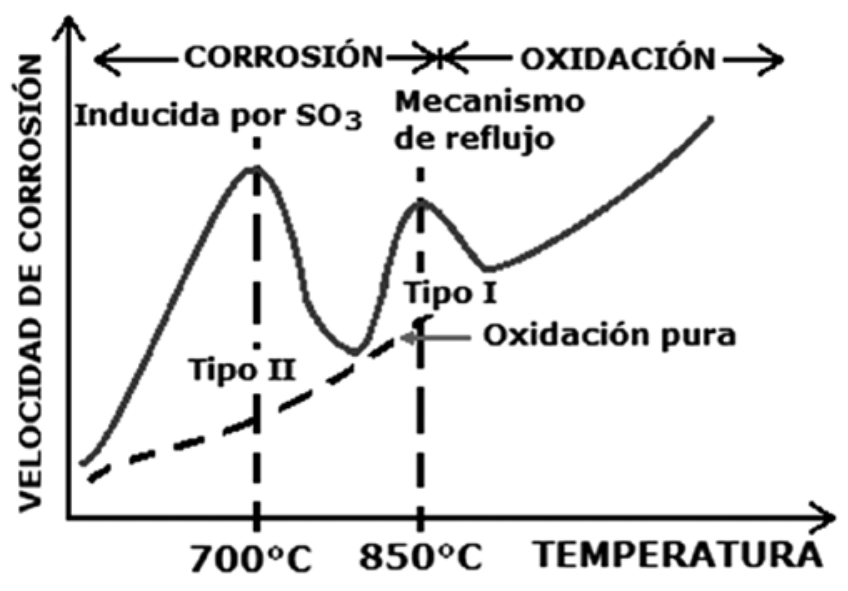

\section{METODOLOGÍA}

Para la realización de los ensayos se fabricaron probetas de forma rectangular de $1 \mathrm{~cm} \times 1 \mathrm{~cm} \times 0.3 \mathrm{~cm}$ de acero laminado en caliente (Hot Rolled). Después, se limpiaron las probetas con disolvente y una vez limpias las probetas, se prepararon superficialmente por lijado manual con papel esmeril número 80, la cual genera una rugosidad de $12 \mu \mathrm{m}$ aproximadamente, y por último se volvieron a limpiar las probetas para dejarlas listas para el rociado térmico. En la tabla 1. Se muestra la composición química del acero de bajo carbono utilizado como sustrato.

Tabla 1. Composición química del acero Hot Rolled. Table 1. Chemical Composition of Hot Rolled Steel.

\begin{tabular}{|c|c|c|c|c|c|c|c|}
\hline $\begin{array}{c}\text { C } \\
\text { (\% en peso })\end{array}$ & $\mathrm{Mn}$ & $\mathrm{Si}$ & $\mathrm{Cu}$ & $\mathrm{P}$ & $\mathrm{S}$ & $\mathrm{Nb}$ & $\mathrm{Fe}$ \\
\hline 0.029 & 0.85 & 0.038 & 0.02 & 0.04 & 0.05 & 0.048 & Balance \\
\hline
\end{tabular}

En la fuente bibliográfica consultada, no se encontró una norma certificada sobre el proceso y aplicación del rociado térmico, por tal motivo para realizar este proceso fue necesario basarse en sugerencias de operación dadas por el fabricante del equipo, por consejos de personal capacitado en aplicación de recubrimientos por de rociado térmico y sugerencias hechas por varios autores consultados $[5,6]$ Para realizar los recubrimientos por de rociado térmico se utilizaron polvos metalúrgicos de acero inoxidable tipo 312 de Eutectic -Castolin, esta aleación contiene pequeñas adiciones de cobre para disminuir su punto de fusión. La aplicación del mismo se realizó utilizando el equipo ROTOTEC (Eutectic - Castolin). El recubrimiento se hizo de acuerdo a las recomendaciones especificadas en el manual del equipo, como las características de la llama: color, tamaño y proximidad con respecto a la pieza, teniendo en cuenta el manejo del combustible (oxígeno-acetileno). Para esta aplicación las presiones que se manejaron fueron de 25 psi para el oxígeno y 11 psi para el acetileno, logrando una temperatura de llama de $3200^{\circ} \mathrm{C}$; se realizó la aplicación del recubrimiento a una distancia de $150 \mathrm{~mm}$ aproximadamente, con un ángulo de aspersión de $90^{\circ}$. Las muestras fueron precalentadas por con llama neutra a 100 ${ }^{\circ} \mathrm{C}$ aproximadamente y las partículas de polvo poseían un tamaño de partícula regular que oscilaba entre 30-90 $\mu \mathrm{m}$. Todas las probetas se rociaron térmicamente durante más tiempo para así darle una capa de recubrimiento más gruesa que la necesaria, para luego, y de forma manual, darle el acabado y las medidas respectivas.

Las sales corrosivas fueron una mezcla sólida de composición $20 \% \mathrm{Na}_{2} \mathrm{SO}_{4}-80 \% \mathrm{~V}_{2} \mathrm{O}_{5}$ (\% en peso), mezcla considerada altamente corrosiva, generada a partir de reactivos de grado analítico. En un mortero se maceraron las sales hasta eliminar grumos y lograr una mezcla homogénea. 
La cantidad de mezcla de sal empleada en cada crisol fue de aproximadamente $7.5 \mathrm{~g}$, utilizando siempre sal fresca para no tener problemas de contaminación. Para evaluar la protección que brinda el recubrimiento aplicado, se hicieron pruebas a diferentes temperaturas para observar cómo se comporta la protección ante estos cambios. Se hicieron pruebas a tres temperaturas: 650,700 y $750{ }^{\circ} \mathrm{C}$, con tiempos de exposición de 1, 7 y 22 horas. Para cada tiempo de exposición se introdujeron dos probetas recubiertas. La limpieza de los productos de la corrosión (decapado) de las probetas se realizó de acuerdo a la norma ASTM G103 "Standard Practice for Preparing, Cleaning, and Evaluating Corrosion Test Specimens" [15], para evaluar la velocidad de corrosión por pérdida de peso.

\section{RESULTADOS}

Los resultados de las pruebas de corrosión se muestran en las figuras 5 y 6 . La figura 5, muestra claramente, que durante la primera hora de exposición los ataques sobre el recubrimiento son aún más severos, que durante los otros tiempos de exposición y a mayor temperatura más agresivo son los ataques por corrosión. La velocidad de corrosión disminuye rápidamente en las primeras horas de exposición y luego tiende a estabilizarse en un valor constante después de un tiempo.

Figura 5. Velocidad de corrosión en función del tiempo, para los aceros recubiertos con el equipo ROTOTEC.

Figure 5. Corrosion rate versus time for the coated steel ROTOTEC equipment.

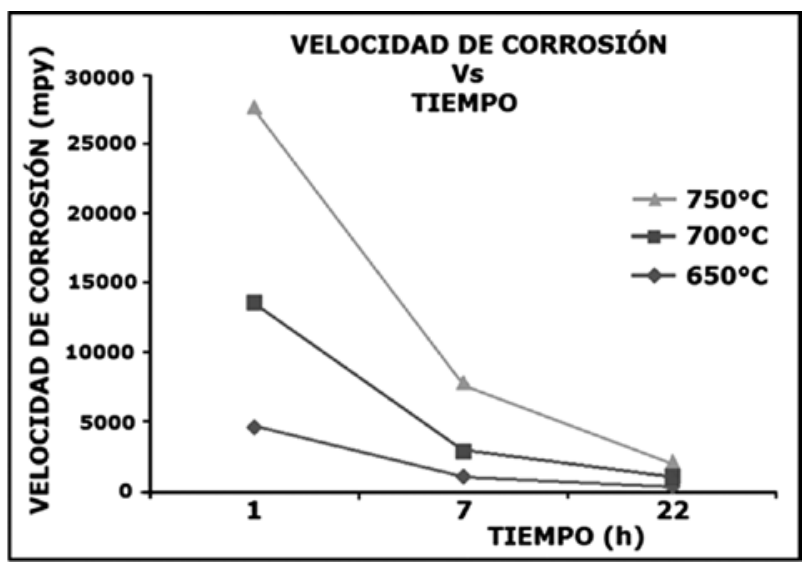

En la figura 6, se observa que al aumentar la temperatura, la velocidad de corrosión se acelera y este cambio es más marcado cuando se pasa de 700 a $750^{\circ} \mathrm{C}$, por esto, la temperatura es un factor muy importante en la degradación del material ya que vuelve líquido el electrolito y acelera el ataque corrosivo del material, esto debido a la formación de ciertos vanadatos complejos de sodio (eutécticos) que disuelven la capa de óxido y aceleran el ataque, debido al aumento de la concentración de los oxidantes activos y su difusividad. Además, como la corrosión se activa térmicamente, esto también se refleja en el aumento de la velocidad de ataque. Se observó el fenómeno de corrosión uniforme sobre el recubrimiento, por la inspección visual antes y después de la limpieza química. Esta corrosión uniforme puede darse por la presencia de una capa de óxido protector y la ausencia de celdas de concentración por diferencia de oxígeno en las caras de las probetas.

Figura 6. Velocidad de corrosión en función de la temperatura, para los aceros recubiertos con el equipo ROTOTEC. Figure 6. Corrosion rates in function of temperature, for the ROTOTEC equipment coated steels.

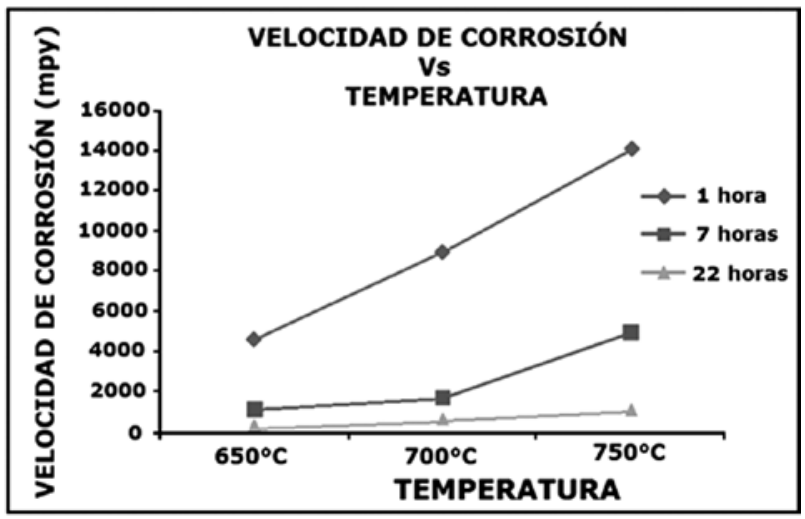

En la figura 7, se observa la velocidad de corrosión de las probetas con recubrimiento y sin recubrimiento a $750{ }^{\circ} \mathrm{C}$. El recubrimiento le brindó protección al substrato, para soportar los ataques corrosivos durante mayor tiempo que las probetas sin recubrimiento, en donde éste actúa como un escudo protector retardando el ataque corrosivo, ya que la corrosión ataca y disuelve el recubrimiento hasta el punto de dejarlo inservible y posteriormente la corrosión empieza atacar al substrato.

Figura 7. Velocidad de corrosión en función del tiempo, para el acero hot rolled recubierto y sin recubrir.

Figure 7. Corrosion rate versus time, for the hot rolled steel coated and uncoated.

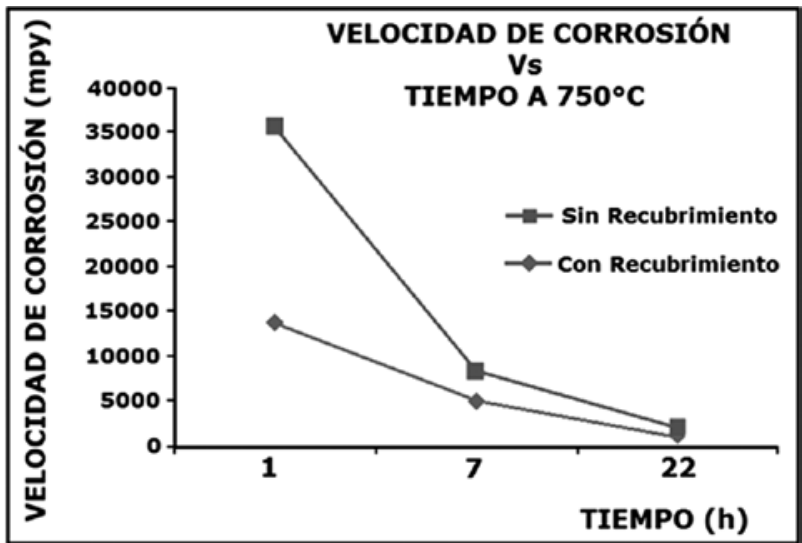


Aunque la corrosión destruye primero a una probeta sin recubrimiento que a otra con recubrimiento, el recubrimiento aplicado no cumple con las expectativas. Por lo anterior, el recubrimiento no realiza un buen desempeño como protección ante la corrosión por sales fundidas de tipo I. Esto indica que el ataque corrosivo depende de la naturaleza de la capa de óxido que se forma en la superficie, de los componentes formados por la aleación y los constituyentes de las sales.

\section{CONCLUSIONES}

- El ataque corrosivo por sales fundidas se incrementa con la temperatura y disminuye con el tiempo de exposición, es decir, la velocidad de corrosión es directamente proporcional a la temperatura e inversamente proporcional al tiempo de exposición.

- Las probetas recubiertas presentaron un tipo de ataque uniforme y óxido adherente al metal base, además el ataque corrosivo disminuyó con el tiempo y tiende a estabilizarse a una velocidad de corrosión más baja que la inicial.

- La velocidad de corrosión de las sales se incrementa con el aumento de la temperatura y es más marcada después de $\operatorname{los} 700^{\circ} \mathrm{C}$, debido a la fusión de ciertos vanadatos de sodio que tienen puntos de fusión bajos, lo que incrementa el ataque corrosivo.

- El recubrimiento rociado térmicamente forma una capa protectora que reduce la velocidad de corrosión, pero no es inmune al ataque corrosivo, por tal motivo no es muy recomendado como sistema de protección contra este fenómeno corrosivo.

\section{AGRADECIMIENTOS}

Los autores expresan sus agradecimientos a la Universidad tecnológica de Pereira por el apoyo al proyecto titulado: Protección contra la corrosión en sales fundidas de un acero por rociado térmico con una aleación níquel cromo.

\section{REFERENCIAS}

[1] Chatha, S., Sidhu, H. \& Sidhu, B. (2012). High temperature hot corrosion behaviour of $\mathrm{NiCr}$ and $\mathrm{Cr} 3 \mathrm{C} 2-\mathrm{NiCr}$ coatings on T91 boiler steel in an aggressive environment at $750^{\circ} \mathrm{C}$. Surface $\mathcal{E}$ Coatings Technology 206 (19-20), 38393850, 2012.
[2] Bradshaw, A., Simms, N. \& Nicholls, J. (2013). Hot corrosion tests on corrosion resistant coatings developed for gas turbines burning biomass and waste derived fuel gases. Surface \& Coatings Technology 228, 248-257, 2012.

[3] Bala, N., Singh, H. \& Prakash, S. (2010). Accelerated hot corrosion studies of cold spray $\mathrm{Ni}-50 \mathrm{Cr}$ coating on boiler steels. Materials and Design 31 (1), 244-253, 2010.

[4] Trevisan, R. \& Lima, C. (2002). Aspersao Termica Fundamentos E Aplicacoes. Sao Paulo: Editorial Artliber.

[5] Kalacska, G., Fazekas, L., Keresztes, R., Toth, A. \& Szepvolgyi, J. (2011). Cold flame-sprayed and oil-impregnated porous metallic coatings. Applied Surface Science 257 (22), 9532-9538.

[6] [7] Davis. J. (2004). Handbook of Thermal Spray Technology. Ohio: Thermal Spray Society and ASM. International.

[8] Marulanda, J., Zapata, A. \& Isaza, E. (2007). Protección contra la corrosión por medio del rociado térmico. Scientia Et Technica 34: 237 - 243.

[9] Marulanda, J. (2006). Fundamentos de la corrosión. Pereira: Universidad Tecnológica de Pereira (UTP).

[10] Sainz, M., Osendi, M. \& Miranzo, P. (2008). Protective $\mathrm{Si}-\mathrm{Al}-\mathrm{O}-\mathrm{Y}$ glass coatings on stainless steel in situ prepared by combustion flame spraying. Surface \& Coatings Technology 202 (9), 1712-1717.

[11] Pawlowski, L. (2008). Finely grained nanometric and submicrometric coatings by thermal spraying: A review. Surface \& Coatings Technology 202 (18), 4318-4328.

[12] Rapp, R. (2002). Hot corrosion of materials: fluxing mechanism? Corrosion science 44 (2): 209-221.

[13] Báez, S., Tristancho, J., Peña, D. \& Vásquez, C. (2004). La espectroscopia de impedancia electroquímica (EIS) aplicada al estudio del mecanismo de la corrosión en caliente por sales fundidas. Dyna, 71, 39-47.

[14] Singh, H., Singh, B. \& Prakash, S. (2006). The role of HVOF coatings in improving hot corrosion resistance of ASTMSA210 GrA1 steel in the presence of $\mathrm{Na}_{2} \mathrm{SO}_{4}-\mathrm{V}_{2} \mathrm{O}_{5}$ salt deposits. Surface \& Coatings Technology 200 (18-19), 5386-5394.

[15] ASTM Standard G1-03. (2003). "Standard Practice for Preparing, Cleaning, and Evaluating Corrosion Test Specimens". ASTM International, West Conshohocken. 